\title{
A Rare Case of 3:1 Alpha Variant
}

\section{Case Report}

Journal of Epilepsy Research pISSN 2233-6249 / eISSN 2233-6257

Received October 29, 2021

Revised November 12, 2021

Accepted November 18, 2021

Corresponding author:

Seolah Lee, MD

Department of Neurology, Seoul National

University Hospital, 101 Daehak-ro,

Jongno-gu, Seoul 03080, Korea

Tel. +82-2-2072-2278

E-mail; seolah920831@gmail.com

\section{Seolah Lee, MD, Sang Kun Lee, MD, PhD}

Department of Neurology, Seoul National University Hospital, Seoul, Korea
Since the first documentation of slow alpha variants in Goodwin et al., there has been a single case report with an actual electroencephalography (EEG). However, any further case has not been reported since then, and neurologists are still unfamiliar with its presence due to its scarcity. Here, we present a rare case of 3:1 subharmonic alpha variant in a hope to acquaint EEG interpretations and speculate upon its benign nature. (2021;11:154-155)

Key words: Alpha rhyth, Electroencephalography, Epilepsy

\section{Introduction}

Normal electroencephalography (EEG), unlike other electrodiagnostic tests, is defined not by having normal components, but by lacking abnormalities. There are numerous patterns that are statistically unusual or rare, but not necessarily abnormal, which complicates the interpretation of an EEG. One such example is alpha variant rhythm. There are fast alpha variants having twice the usual frequency or slow alpha variants with half the frequency. However, in rare cases as Goodwin ${ }^{1}$ pointed out, alpha variants can have 3:1 or 4:1 ratio compared to the basic rhythm. In 2012, there was a case report $^{2}$ of 3 for 1 subharmonic alpha rhythm with actual EEG images; but after this report, we could not find any related articles, especially in Korea. In this study, we report a patient with unique 3 for 1 slow alpha variant for better recognition of this benign variant.

\section{Case Report}

A 56-year-old female visited neurologist for recurrent loss of consciousness. She complained of having fits of hyperventilation and loosing awareness, sometimes accompanied with numbness around her left face. Her autonomic function test revealed having a postural orthostatic tachycardia syndrome. The first impression was panic disorder, but in order to rule out epilepsy, she underwent routine EEG using standardized 10-20 international system. Her EEG showed nearly continuous run of 3-3.5 Hz notched delta activities in both oc- cipital to posterior temporal regions (Fig. 1). This pattern was blocked by eye opening and restored with eye closure, but only after a brief preceding alpha rhythm of $9 \mathrm{~Hz}$. However, photic stimulation did not bring about the blocked pattern nor did it evoke any photic driving response. When the 25-min EEG was taken, the $3 \mathrm{~Hz}$ notched pattern predominated her posterior dominant rhythm for almost 20 minutes, while it did alternate with a few periods of $9 \mathrm{~Hz}$ alpha rhythm. Besides this unique background rhythm, her EEG was insignificant without any signs of epileptiform discharge or slow activity.

\section{Discussion}

By the definition revised in the standard clinical care EEG terminology, $^{3}$ the pattern shown in this EEG can be classified as rhythmic delta activity. It is clearly rhythmic, with repetition of waveform of uniform morphology and duration, and of delta range. However, considering the benign nature of the pattern, we might safely call it a benign alpha variant. First, the pattern is reactive to eye opening and closure. Second, it is only restricted in the occipital area and shows no sign of spatial evolution. Lastly, it alternates with $9 \mathrm{~Hz}$ alpha rhythm, which indicates that the pattern is subharmonic variant of $9 \mathrm{~Hz}$ rhythm. The pattern is also distinguished from occipital intermittent rhythmic delta activity (OIRDA), which does not have a uniformly notched appearance. Unlike the case previously reported in $2012{ }^{2}$ which only had few seconds of variant rhythm and then returned back to $9 \mathrm{~Hz}$ background, our case showed nearly $90 \%$ preva- 


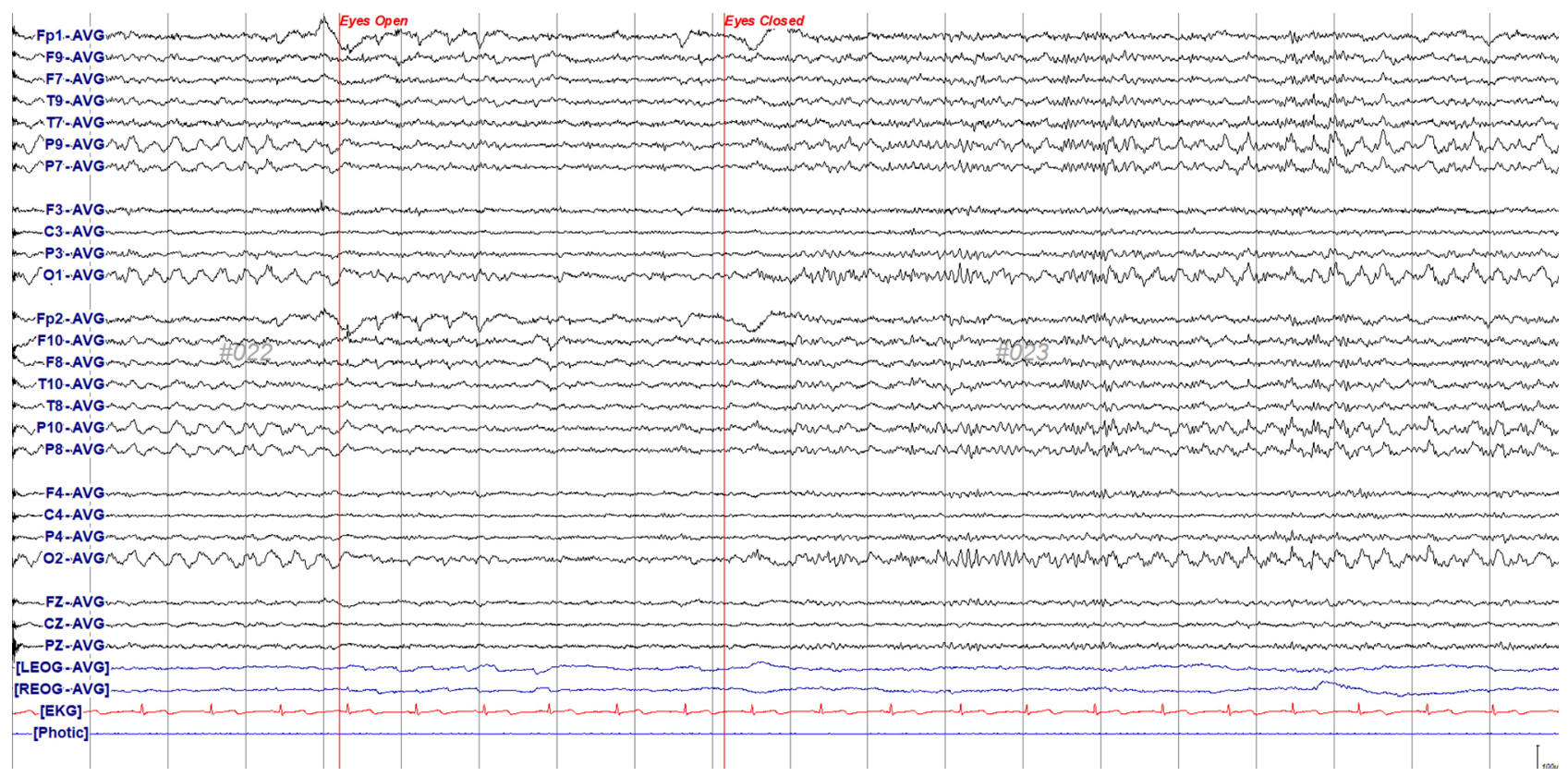

Figure 1. Standard 10-20 international system electroencephalography showing 3 for 1 alpha variant that is blocked by eye opening and restored after preceding alpha rhythm following eye closure.

lence of the slow variant over the usual alpha rhythm and even in fully wakeful periods. This prevalence makes us believe that $3 \mathrm{~Hz}$ variant rhythm is her background activity. Yet the clinical significance is unknown, we find it important to recognize this particular type of rhythmic delta activity as benign.

\section{Conflict of Interest}

The authors declare that they have no conflicts of interest.

\section{Acknowledgements}

Sang Kun Lee is the editor in chief of the Journal of Epilepsy Research.

\section{References}

1. Goodwin JE. The significance of alpha variants in the EEG, and their relationship to an epileptiform syndrome. Am J Psychiatry 1947;104: 369-79.

2. Beauchemin P, Savard M. Slow alpha variant: a report of a 3 for 1 ratio subharmonic. Can J Neurol Sci 2012;39:97-8.

3. Hirsch $\sqcup$, Fong MMK, Leitinger $M$, et al. American Clinical Neurophysiology Society's Standardized Critical Care EEG Terminology: 2021 version. J Clin Neurophysio/ 2021;38:1-29. 